\title{
Anticipating who will say what: The influence of speaker-specific memory associations on reference resolution
}

\author{
William S. Horton • Daniel G. Slaten
}

Published online: 5 August 2011

(C) Psychonomic Society, Inc. 2011

\begin{abstract}
Some accounts of common ground assume that successful communication requires detailed consideration of others' knowledge. In two studies, we provide evidence for an alternative account that views common ground as being mediated in part through domain-general memory mechanisms. On each trial, participants heard prerecorded instructions from one of two speakers indicating which of two displayed pictures to select. During an initial association phase, each speaker repeatedly referred to different sets of pictures. In Experiment 1, we contrasted a "betweenspeaker mapping" condition, in which each speaker referred to only one picture from critical item pairs (e.g., the cat drinking milk vs. the cat sitting up), and a "within-speaker mapping" condition, in which each speaker referred to both pictures within each pair, although item categories differed across speakers. On subsequent test trials, we recorded participants' eye fixations to critical displays that included both items from a category pair. Prior to the linguistic point of disambiguation, participants in the between-speaker mapping condition were more likely to fixate on the picture previously described by the current speaker, suggesting that knowledge associated with the speaker was prompting expectations for which picture would be the intended target. In Experiment 2, we used two prerecorded speakers of the same gender to strengthen the claim that the relevant implicit memory associations are speaker-specific. These results demonstrate how domain-general memory associations can be an important constraint upon language use.
\end{abstract}

W. S. Horton $(\bowtie) \cdot$ D. G. Slaten

Department of Psychology, Northwestern University,

2029 Sheridan Road,

Evanston, IL 60208-2710, USA

e-mail: whorton@northwestern.edu
Keywords Common ground · Reference resolution . Memory Eyetracking

A central assumption in conversational pragmatics is that language use is shaped by beliefs about common ground. That is, inferences about shared knowledge are seen as critical for allowing speakers to design utterances for addressees, and for allowing addressees to understand speakers' intentions (Clark, 1996; Clark \& Carlson, 1981). For example, in one series of studies, Brennan and Clark (1996) showed that, once interlocutors have jointly settled upon specific labels for particular objects, speakers will continue to use these expected referring expressions even when the communicative context changes in ways that make such expressions overly specific. To account for this phenomenon, Brennan and Clark proposed that interlocutors establish mutually agreed-upon conceptualizations of objects known as conceptual pacts, which constrain the form and content of subsequent references to the same objects (see also Van der Wege, 2009). The existence of such pacts in common ground also helps to explain why deviations from previously established expressions can interfere with listeners' identification of specific referents (Metzing \& Brennan, 2003).

In contrast, Barr and Keysar (2002; see also Kronmüller $\&$ Barr, 2007) have suggested that language users are primarily sensitive to the simple availability of previously used labels (i.e., linguistic precedents) and that these labels are not necessarily partner specific. Thus, when suitable precedents exist, individuals are prepared to accept expressions consistent with such precedents, regardless of their source. Indeed, in several experiments, Barr and Keysar (2002) found that reference resolution was influenced more by the availability of referential precedents than by the identity of the speaker with whom such precedents were 
established. On this view, language users consider representations of shared knowledge only as necessary to update the initial products of early, egocentric processes (Keysar, Barr, Balin, \& Brauner, 2000). When linguistic precedents are highly available, processing can occur on the basis of precedents alone, without consideration of whether those precedents are part of common ground.

\section{Memory-based processing and language use}

As this brief overview makes clear, debates in this area have frequently centered on the essential versus nonessential role of common ground in language use. An equally important goal, though, involves clarifying the nature of the cognitive mechanisms that potentially constrain when and how language users interact on the basis of assumptions about common ground. As a step toward this goal, Horton and Gerrig (2005a) outlined an account of conversational common ground rooted in basic processes of memory encoding and retrieval. On this account, other interlocutors can become linked, in a low-level fashion, to a wide range of contextual information via ordinary processes of associative encoding. Once established, these person-specific associations allow those same individuals to function as salient cues for the subsequent retrieval of this information from long-term memory. Similar to memory models that posit cue-based access to stored memory traces (e.g., Gillund \& Schiffrin, 1984; Medin \& Schaffer, 1978; Ratcliff, 1978), this automatic retrieval takes place through a mechanism known as resonance (cf. Grossberg, 1980), which serves to increase the accessibility of memories associated with available retrieval cues. For conversational contexts, we argued that if the cues related to a specific interlocutor boost the accessibility of associated information past some threshold, this has the potential to constrain language processing in ways that are consistent with pragmatic expectations about common ground. Importantly, though, resonance is not a goal-driven process. That is, this memory retrieval can occur on the basis of any set of contextual cues that share sufficient overlap with the original encoding context. For purposes of common ground, the most influential cue-driven constraints may be anything that happens to be strongly associated with specific interlocutors in particular conversational contexts.

A variety of work has documented the impact of this kind of cue-driven memory retrieval in other language processing contexts. For example, memory-based approaches to text comprehension have demonstrated how textual cues can drive the low-level activation of related concepts in ways that facilitate the resolution of syntactic ambiguities (Rawson, 2004), promote relevant inferences (McKoon \& Ratcliff, 1992, Myers \& O'Brien, 1998), and serve to bind disparate parts of a text together into coherent wholes (Gerrig \& McKoon, 2001; Myers, O’Brien, Albrecht, \& Mason, 1994). In one series of studies, Greene, Gerrig, McKoon, and Ratcliff (1994) presented participants with stories about two characters who shared an initial interaction but then separated. Measuring probe recognition latencies, Green et al. found that concepts mentioned as part of the initial interaction (e.g., "cousin") became accessible again when the characters reunited in the story. Subsequently, Lea, Mason, Albrecht, Birch, and Myers (1998) showed that this information did not have to be part of the characters' common ground; even when initially privileged to one character (e.g., one character thinking to herself about the cousin), the accessibility of target concepts increased after the characters reunited. To explain these reunion effects, one can assume that bringing the characters together again coactivated related concepts for readers in ways that resonated with previously encoded information sharing some degree of lexical or conceptual overlap (although see Trabasso \& Wiley, 2009, for an alternative account).

Further research has provided evidence consistent with a role for memory-based processing in how speakers access information about common ground during language production (Horton, 2007; Horton \& Gerrig, 2005b). In these studies, participants engaged in an initial set of interactions with each of two experimental partners, followed by a task designed to examine how the memories encoded as part of those initial experiences would shape subsequent language use with the same partners. For example, Horton and Gerrig (2005b) used a card-sorting task to show that the specificity of the memory traces established with respect to each conversational partner influenced speakers' later success at audience design, whereas Horton (2007) demonstrated that initial partner-specific memory associations could facilitate picture naming in the context of the same partners, even in the absence of an overt communicative goal.

The aim of the present work was to extend these findings by examining the influence of speaker-specific associations during spoken message interpretation and, specifically, to investigate how the nature of the memory associations established between specific speakers and particular conversational referents can shape processes of real-time reference resolution. In doing so, this will provide additional support for the claim that domain-general processes of memory encoding and retrieval can mediate important aspects of language use.

\section{Experiment 1}

Similar to Horton and Gerrig (2005b) and Horton (2007), in the present experiments, we used an initial association 
phase to establish specific mappings between referents and each of two speakers, followed by a test phase that examined how these speaker-specific associations affect referent identification in the context of each speaker subsequently. On each trial, participants heard prerecorded instructions from one of two speakers to click on one of two pictures presented via computer. During the test phase, we examined the time course with which participants considered possible referents by recording eye fixations during critical utterances that were temporarily ambiguous. For example, in the context of a display containing two cats, one sitting upright and the other drinking milk, an utterance such as "Click on the cat..." is ambiguous until additional information is provided, such as "...that's sitting up." Thus, critical items were created in pairs, with the items in each pair differing only in the relevant disambiguating information (e.g., "sitting up" vs. "drinking milk").

We are interested in whether listeners can use information about speaker identity to anticipate likely referents even before the speaker has disambiguated them linguistically. To vary the usefulness of speaker identity as an early cue for reference resolution, the association phase involved one of two possible mappings between speakers and critical items. As shown in Fig. 1a, participants in the betweenspeaker mapping condition heard each speaker, over different blocks of trials, repeatedly refer to only one item from a given item pair. The goal of this condition was to associate each speaker with a specific item from each pair (along with an appropriate referring expression). As shown in Fig. 1b, though, participants in the within-speaker mapping condition heard each speaker refer to both items from each pair, and item categories differed across speakers. The goal of this mapping was to associate each speaker with both items (and their referring expressions) for certain pairs. Importantly, participants always saw a given item with its associated label the same number of times, regardless of condition. Thus, the critical difference between conditions lies in the nature of the speakerspecific mappings with particular items and item pairs.

In the test phase, both items from each experimental pair appeared together on critical trials. Importantly, the intended target of the spoken referring expression could either be the item to which the current speaker had referred multiple times previously - the same speaker condition - or it could be the picture to which the other speaker had always referred previously - the different speaker condition. Varying whether critical expressions were produced by the same or by a different speaker permits examination of the effect of speaker-specific information on reference resolution. Following the within-speaker mapping, when both items on test displays are associated with the current speaker (or with the other speaker), the speaker alone
Fig. 1 Example experiment organization for the association phase and test phase of a the between-speaker mapping condition (Experiments 1 and 2), and b the within-speaker mapping condition (Experiment 1 only). In the test phase, two possible combinations of same- and different-speaker trials are shown a

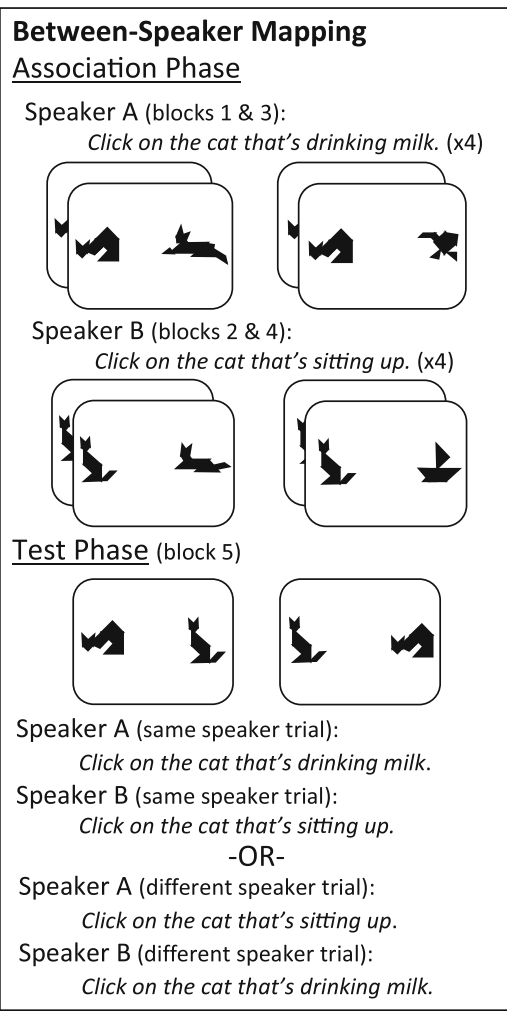

b

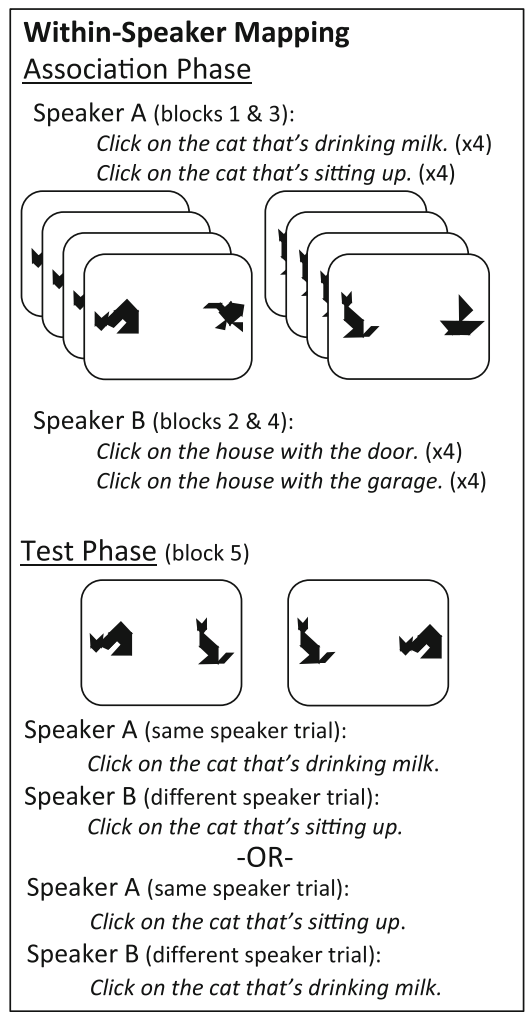


should not prime listeners to expect one referent over another. However, when only one item is uniquely associated with a current speaker, as is the case following the between-speaker mapping, listeners may implicitly anticipate that the associated item will be the target.

Unlike Barr and Keysar (2002) and Metzing and Brennan (2003), both of which utilized conditions in which referring expressions for critical objects were initially established with one speaker (the second speaker was someone completely "new"), in the present study, we examined a situation in which both speakers were associated with specific object-expression mappings, which we then pitted directly against each other. Given the memorybased account, we predicted that listeners would use speaker identity as a retrieval cue and show early preferences for looking at items associated with the current speaker-but only when suitably distinct speaker-specific mappings are available.

\section{Method}

\section{Participants}

Forty-one undergraduates participated in this experiment for course credit or monetary compensation. Nine participants were excluded because of failure to follow directions or poor calibration. All participants were native English speakers.

\section{Materials}

Experimental items consisted of 32 tangrams, organized into 16 item pairs created to resemble specific object types. Both members of an item pair belonged to the same category, such as "rabbits" or "women." For each item, we generated a plausible referring expression consisting of a head noun plus a post-nominal modifier. Expressions for critical pairs involved the same head noun but different modifying information, such as rabbit that has two ears and rabbit that's sitting down. See the Appendix for the complete list of critical item labels. For each experimental pair, two additional Tangrams from the same category served as foils on initial trials. These foils lacked identifying features mentioned in critical expressions.

Filler items consisted of 16 sets of black and white line drawings of everyday objects. Each set included four pictures from the same object category that, like experimental pictures, contrasted on specific features (e.g., watches with or without stripes on the wristband). We generated referring expressions for filler pictures similar to expressions used for experimental items, except that many involved prenominal modification (e.g., blank watch). All pictures were scaled to approximately $200 \times 200$ pixels in size, and two pictures were presented on each display. The pictures were positioned to the left and right of the center of the screen, at roughly 6 degrees of visual angle relative to the viewing position of the participant. We balanced the locations of targets equally across trials.

For each display, we recorded instructions from two speakers. In Experiment 1, we used one male speaker and one female speaker to render the voices maximally distinct. The instructions were scripted to convince participants that speakers were naive participants. For example, utterances from early blocks were longer and contained disfluencies that gradually disappeared from later utterances. The instructions were digitally recorded in a sound-attenuating booth at a $22.05-\mathrm{kHz}$ sampling rate.

Association phase The association phase consisted of four blocks of trials. All instructions in each block came from a single speaker, and speakers alternated across successive blocks. Each block included 32 experimental and 32 filler trials. The 32 experimental trials in a given block included 16 experimental items as targets, which were presented twice with different distractors across presentations. These items were randomized within each half block, such that participants saw a full set of 16 experimental items before the same pictures appeared a second time. In both speakermapping conditions, 16 of the experimental items appeared during blocks with one speaker, and the other 16 experimental items appeared during blocks with the other speaker.

Items from critical pairs always appeared on separate trials in the association phase. During the first two blocks, experimental items were presented with same-category distractors to help motivate the use of specific referring expressions. For example, the item referred to as the man who's running would appear with another Tangram depicting a man standing. In the next two blocks, critical targets appeared with unrelated distractors that were assigned to displays by switching distractors across item categories. Filler items followed the same patterns. In addition, filler trials often involved targets that had been distractors on previous trials. This informed participants that speakers would sometimes refer to objects they had not described previously, making the different-speaker trials of the test phase somewhat less unexpected.

We created two versions of the association phase, one for each mapping condition. Participants in the betweenspeaker mapping condition heard each speaker repeatedly refer to one item from each of the 16 experimental item pairs, with different items across speakers. For example, the male speaker would refer to the cat that's sitting up, and the female speaker would refer to the cat that's drinking milk. Participants in the within-speaker mapping condition heard each speaker describe, on separate trials, both members of 
experimental item pairs, and item categories differed across speakers. For instance, the male speaker might repeatedly describe both cats, whereas the female speaker described both houses. Figure 1 provides a schematic example of how the two mapping conditions were organized. In both conditions, each experimental item was ultimately described four times by one of the two speakers during the association phase. Thus, following the between-speaker mapping, each speaker became uniquely associated with one item from each of the 16 experimental pairs, or, following the within-speaker mapping, with both items from eight pairs.

Test phase The test phase consisted of 32 experimental and 32 filler trials. Importantly, the 16 critical trials presented both items from critical item pairs (e.g., the sitting rabbit and the two-eared rabbit). In addition, there were 16 control trials that included an experimental item as the target (the sitting rabbit) with a different-category competitor (the standing man). Similarly, there were 16 filler trials that involved targets paired with a same-category item and 16 filler trials that included different-category distractors.

Participants heard each speaker give instructions for 32 (16 experimental, 16 filler) of the 64 trials presented during the test phase. Unlike the association phase, trials from both speakers were randomly intermixed, which forced participants to attend to speaker identity on a trial-by-trial basis. Finally, half of the experimental trials involved targets that had been previously identified by the same speaker (same speaker condition), whereas the remaining trials involved targets previously identified by the other speaker (different speaker condition). For example, if the male speaker had previously described the cat that's sitting up and described it again in the test phase, this would be a same-speaker trial. However, if the female speaker had previously described this item, this would be a different-speaker trial. Speaker identity was counterbalanced across item pairs across versions of the experiment. Figure 1 illustrates, for both mapping conditions, the manipulation of speaker identity during the test phase.

\section{Procedure}

The cover story stated that classmates had taken part in a communication study that involved describing simple objects for unknown listeners. The participants were told that they would be hearing instructions recorded independently by two previous speakers, one male and one female. The cover story emphasized that the recordings were taken from the exact sequences experienced by those individuals, which was intended to make clear that each speaker saw a unique set of items with no access to the items or descriptions from the other speaker.
Each trial consisted of the following sequence: a central fixation point appeared for $1.5 \mathrm{~s}$, followed by a "preview" of the two pictures for $2 \mathrm{~s}$, then a fixation point again for $2 \mathrm{~s}$, and finally the two pictures reappeared simultaneously with the onset of the auditory instructions. We included the preview to allow listeners to encode both pictures prior to hearing the actual instruction. After participants selected a picture on each trial, the next trial began.

During the first two blocks of the experiment, participants simply followed instructions from the speakers. Before the third block, participants were taken through a brief calibration procedure for the eyetracker and were recalibrated again before blocks four and five. Eye fixations were recorded using an ASL 6000 desk-mounted eyetracker with GazeTracker software. Following the test block, participants were debriefed concerning the purpose of the experiment. All 32 participants indicated that they had believed that the instructions were from naive participants.

\section{Design}

The nature of the speaker-specific mapping during the association phase (between vs. within) was a betweensubjects factor, whereas the speakers' relationship to targets in the test phase (same vs. different) was a within-subject factor. The presence of same- vs. different-category competitors on test trials was also manipulated within subjects, although our analyses focus on data from trials with samecategory competitors. These factors were counterbalanced across eight versions of the experiment. Each experimental item appeared as the target once during the test block. Items were also counterbalanced to ensure that each item appeared in all conditions across versions.

\section{Results}

We coded the locations of participants' eye fixations during each trial, starting at the onset of the speaker's instruction and ending when participants clicked on a screen location to end the trial. Each fixation was coded as landing on the intended target of the speaker's referring expression, on the competitor object, or on neither location. Then, for experimental trials involving same-category competitors, we calculated the proportions of total fixations to the intended target and the proportions of total fixations to the competitor. ${ }^{1}$ To provide a sense of the overall manner in which participants' attention shifted toward the target as the instructions unfolded, in

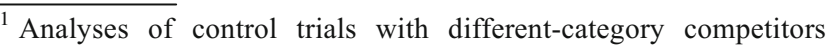
showed early disambiguation effects, such that listeners showed strong preferences for looking at targets immediately after the onset of the head noun, but this did not vary across speaker or mapping conditions.
} 
Fig. 2 we present the proportions of fixations to the target for the within-speaker and between-speaker mapping condition, grouped into 100-ms bins centered on the onset of the head noun. As this figure shows, participants generally waited for disambiguating information before looking strongly to the target, particularly in the within-speaker mapping condition. For the between-speaker mapping condition, however, speaker identity appeared to influence looking behavior such that, before the point of disambiguation, listeners were more likely to look at the target on same-speaker trials than on different-speaker trials.

To analyze these patterns, we identified three temporal windows in each trial, time locked to the accompanying utterance, as indicated in Fig. 2. These window boundaries take into account the fact that approximately $200 \mathrm{~ms}$ are required to plan and execute a saccade, and also that critical postnominal modifiers began, on average, $790 \mathrm{~ms}$ after the head noun. Thus, the baseline period consisted of fixations prior to $200 \mathrm{~ms}$ following the onset of the head noun, before any useful speech had occurred, whereas the predisambiguation period began at $200 \mathrm{~ms}$ and extended until approximately $990 \mathrm{~ms}$ following noun onset, reflecting the period when only the head noun had been heard. Finally, a postdisambiguation period began at $990 \mathrm{~ms}$ and lasted until a 1,700 ms postnoun onset (or the point at which participants selected a picture, whichever came first), indicating the region when listeners had heard enough information to identify the correct referent. Within each period, we computed a "target advantage score" by subtracting the proportions of looks to the competitor (out of all looks during each time period) from the proportions of fixations to the target (again, out of all looks during each time period). The target advantage score reflects the relative preference for looking at the target as the utterance unfolds, and the mean scores across periods and conditions are presented in Fig. 3. We carried out ANOVAs on target advantage scores for each time region separately, treating both subjects $\left(F_{1}\right)$ and items $\left(F_{2}\right)$ as random variables.

In the baseline period, there were no differences among conditions (all $F_{\mathrm{S}}<1.1$ ). Before hearing the referring expression, listeners showed an equal preference for looking at the target versus the competitor. In the critical predisambiguation period, however, there was a reliable interaction between speaker mapping and speaker identity, $F_{1}(1,30)=$ $11.47, M S E=.037, p<.01 ; F_{2}(1,31)=7.50, M S E=.123$, $p<.01$. The mean target advantage score was significantly higher for same speaker trials than for different speaker trials for the between-speaker mapping, $t_{1}(15)=3.37, p<.01$; $t_{2}(31)=3.41, p<.01$, but the same comparison for the within-speaker mapping was not significant, $t_{1}(15)=$ $1.29, p=.22 ; t_{2}(31)=1.01, p=.32$. Thus, listeners looked more strongly at the target when it was associated with the

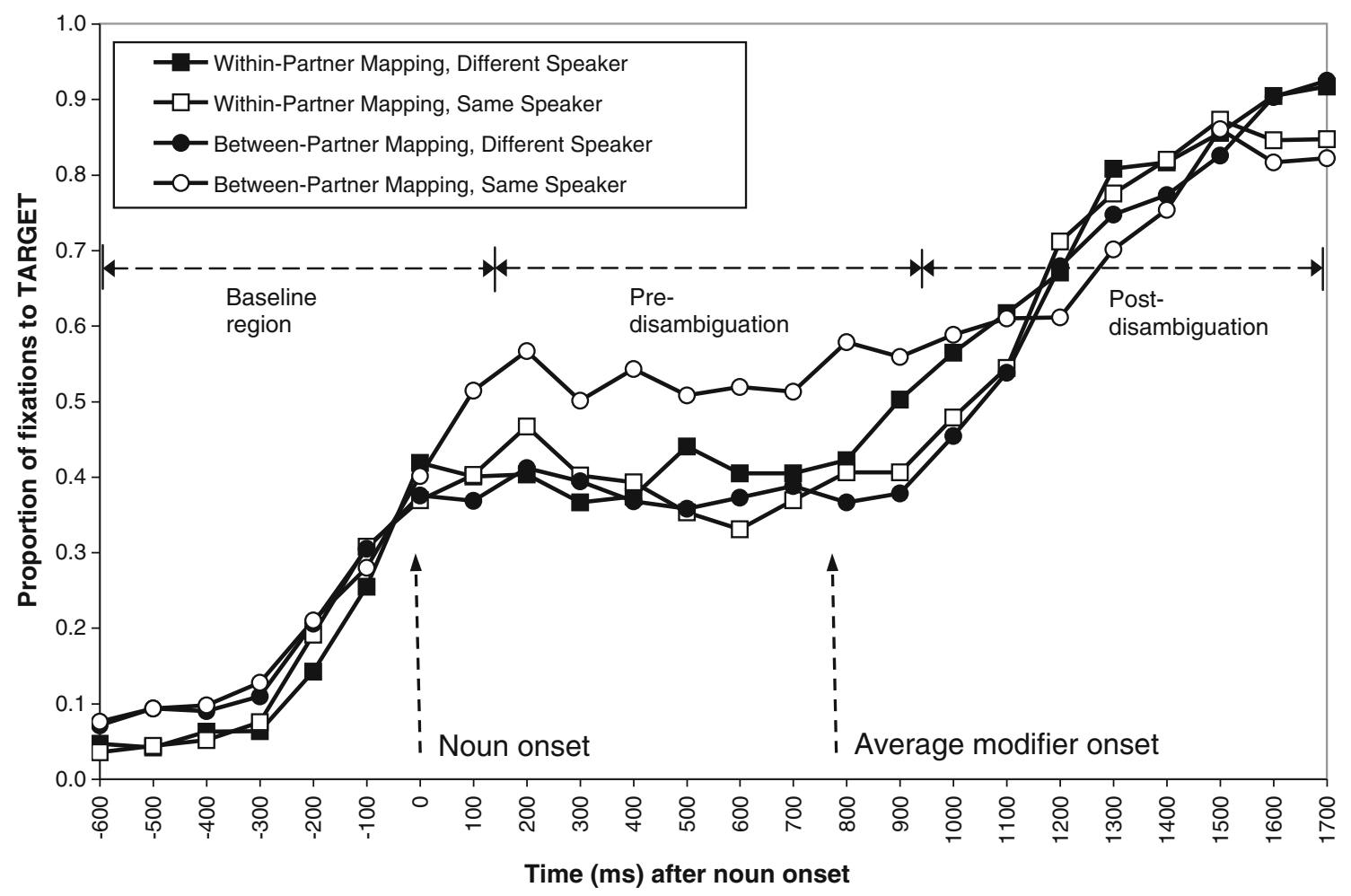

Fig. 2 Mean proportions of fixations to the target on experimental trials in the four conditions in Experiment 1, calculated in 100-ms bins. Trials are aligned to the onset of the head noun, for example, "man," at 0 ms. The average onset of the critical postnominal modifier, for example, "running," is at $790 \mathrm{~ms}$. Average regions of analysis are also marked 
Fig. 3 Mean target advantage scores for the between-speaker mapping and within-speaker mapping conditions in Experiment 1 , by speaker identity and analysis regions time-locked to the accompanying speech. The target advantage score represents the proportion of total fixations to the target minus the proportion of total fixations to the competitor during each region. Error bars represent standard errors of the means for each group

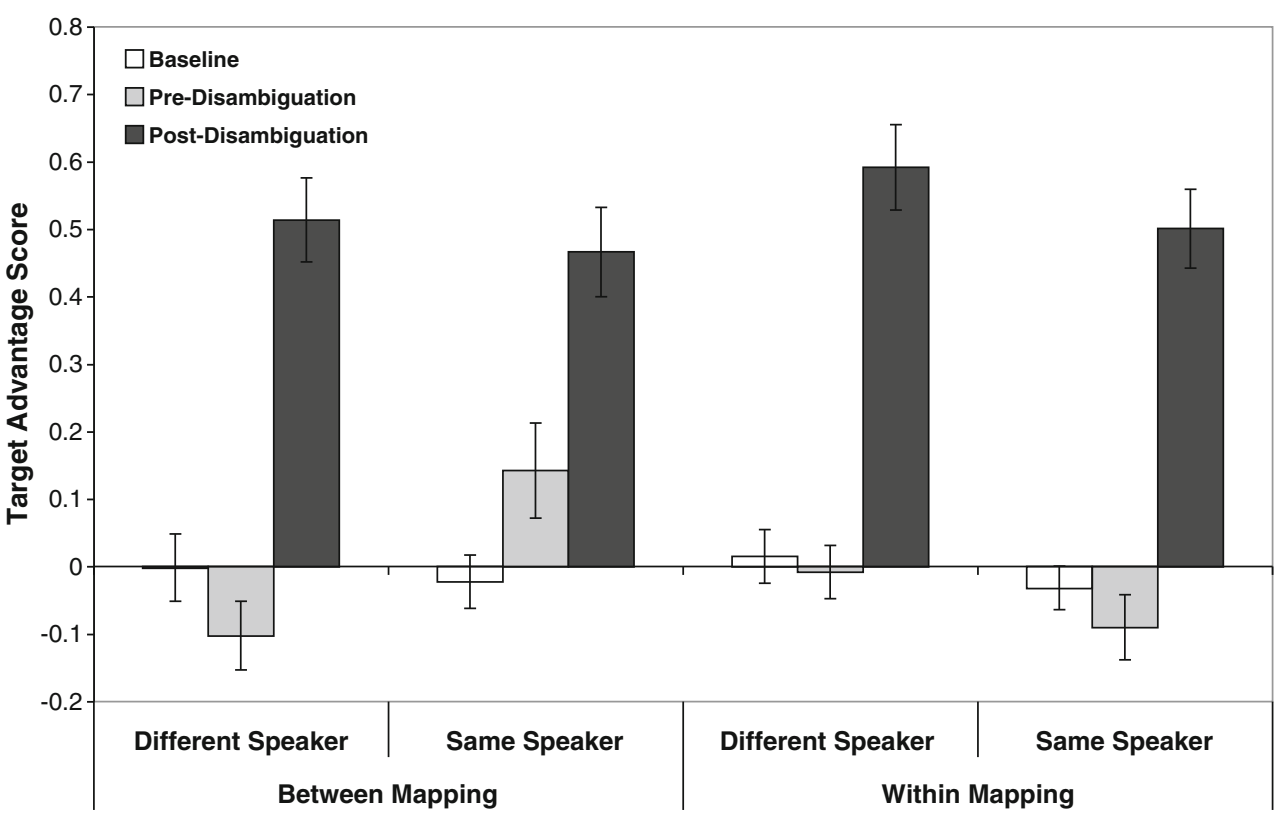

current speaker, but only when each speaker was associated with a unique item in the display. Indeed, the pattern of fixation proportions for the predisambiguation period in Fig. 3 shows that although listeners in the between-mapping condition looked more at targets on same-speaker trials [target advantage score significantly greater than zero: $t_{1}(15)=$ $\left.2.02, p<.05 ; t_{2}(31)=1.81, p<.05\right]$, they looked more at competitors on different-speaker trials [target advantage score significantly less than zero: $t_{1}(15)=-1.99, p<.05$; $\left.t_{2}(31)=-1.59, p=.06\right]$. Recall that on different-speaker trials, the current speaker was associated with the competitor, not the target. Thus, prior to the point of disambiguation, listeners in the between-speaker mapping condition tended to look at the picture associated with the current speaker-even if that picture ultimately turned out to be "wrong." Finally, in the postdisambiguation period, listeners overwhelmingly looked at the target, which by that time had been fully identified. As in the baseline region, there were no differences among conditions (all $F \mathrm{~s}<1.3$ ).

The different-speaker trials, in which the current speaker referred to the picture that he or she had not described previously, were not consistent with the associations established for that speaker and the other picture in the display. One implication of the memory-based account, though, is that associations in memory are subject to revision as individuals encounter new information. Thus, exposure to trials containing unexpected referring expressions may weaken existing mappings between the same referents and the other speaker. To examine how this may have influenced anticipatory looks to the potential referents of particular expressions, we carried out a final analysis in which we split the experimental data from the betweenspeaker mapping condition into those that occurred either early (first half) or late (second half) during the test phase. Figure 4 plots the mean target advantage scores for these early versus late trials, separated by temporal region and speaker condition. Focusing on the critical predisambiguation period, there is an apparent effect of trial position, such that the difference in target advantage scores across speaker conditions is stronger for earlier trials than for later trials.

To verify this pattern, we analyzed target advantage scores in the predisambiguation period for the between-speaker mapping condition, with trial position (early vs. late) and speaker identity as factors. However, because item order during the test phase was determined randomly, individual items did not appear equally across all conditions in both halves of the test phase, leading to numerous empty cells when mean target advantage scores were computed on a peritem basis. Thus, we report only the results of the ANOVA by participants, which revealed a marginally significant Trial Position $\mathrm{x}$ Speaker interaction for the predisambiguation period, $F_{1}(1,15)=4.10, M S E=.122, p=.06$. Pairwise comparisons revealed that the difference in target advantage scores across speaker conditions was significant for early trials, $t_{1}(15)=3.24, p<.01$, but not for late trials, $t_{1}(15)=$ $.87, p=.40$. These patterns suggest that, after experiencing early trials in which speakers referred to unexpected pictures, listeners were less likely to use speaker identity to anticipate reference on later trials when the same referents appeared again.

\section{Discussion}

In Experiment 1, participants were given the opportunity to encode associations between specific items, their labels, 
Fig. 4 Mean target advantage scores for early and late experimental trials in the betweenspeaker mapping condition only in Experiment 1, by speaker identity and analysis regions time-locked to the accompanying speech. The target advantage score represents the proportion of total fixations to the target minus the proportion of total fixations to the competitor during each region. Error bars represent standard errors of the means for each group

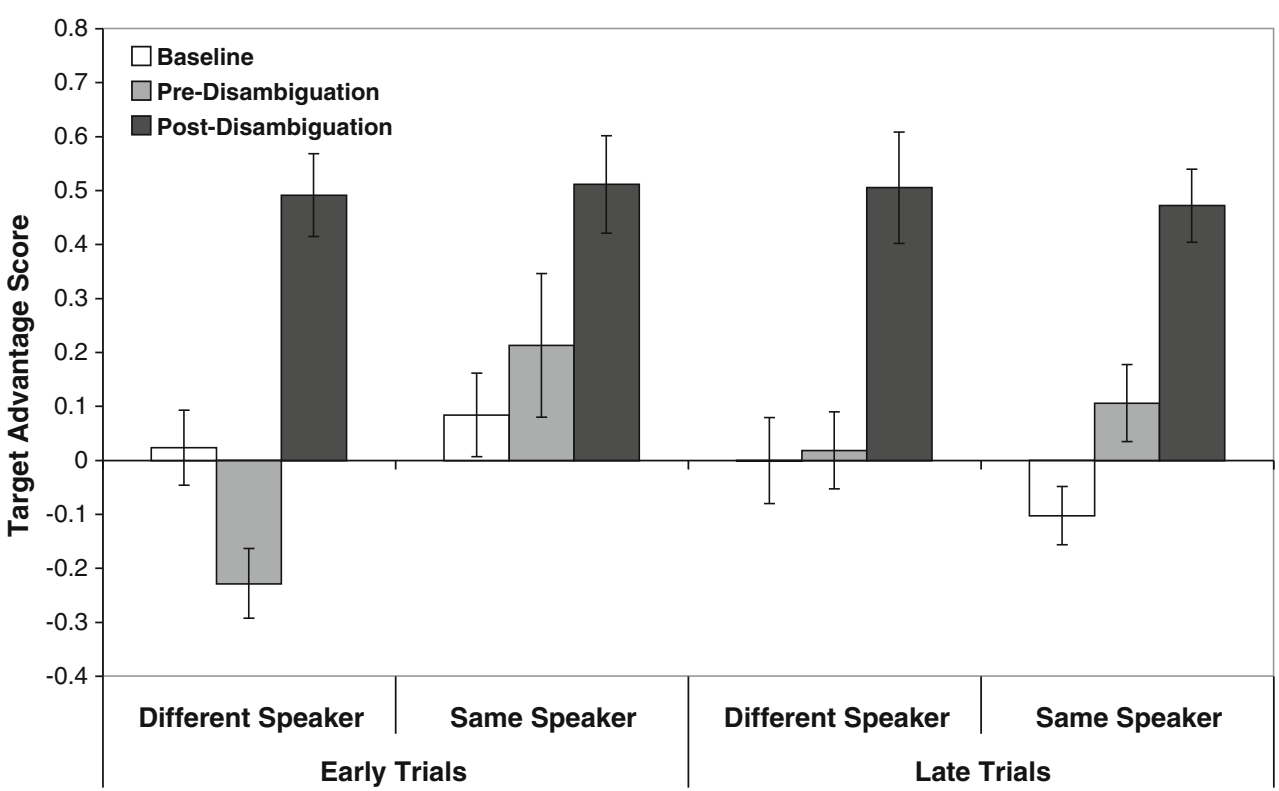

and each of two prerecorded speakers. Subsequently, on test trials in which items from critical pairs were presented together, participants were more likely to look at the item associated with the current speaker, even before the target referent was fully disambiguated-but only when each speaker was uniquely associated with one of the two items. Moreover, the apparent influence of these speaker-specific memory associations weakened over the course of the test phase, presumably as participants experienced trials in which speakers referred to referents in ways that mismatched prior associations (Brown-Schmidt, 2009). These results support the memory-based account of language use (Horton \& Gerrig, 2005a). Speaker-specific cues enabled associated information to become accessible in ways that constrained early reference resolution.

In Experiment 1, we used one male and one female speaker to create distinct mappings across items and referring expressions. We did this to reduce any ambiguity about which individual was acting as the speaker on a given trial. This fact, however, raises a question about the source of participants' apparent sensitivity to speaker-specific cues in the between-speaker mapping condition. That is, it is possible that the observed patterns of early looks to expected targets were driven by distinct mappings at the level of gender rather than through specific associations with individual speakers. This possibility is supported by Senay and Keysar (2009), who found that listeners could use speakers' gender as a cue to constrain referential interpretation. Although this would still be consistent with one implication of the memory-based account, which is that comprehenders likely rely on a range of available information to constrain language understanding, including anything from the communicative context that is highly correlated with particular meanings, it is still important to explore whether listeners can anticipate reference on the basis of the identities of individual speakers (and not just via semantic properties such as gender), given that such speaker-specific constraints are potentially an important facet of many conversational contexts. Thus, in Experiment 2 , we carried out a replication of the previous study that used as communicative "partners" two prerecorded speakers of the same gender.

\section{Experiment 2}

Experiment 2 followed the same logic as Experiment 1 . Following an association phase intended to establish speaker-specific associations in memory for particular object-expression pairings, we recorded the eye movements of participants as they carried out a set of test trials in which the same two speakers referred to critical objects in ways that were either consistent or inconsistent with these previous associations. Critically, the two individuals serving as prerecorded speakers were both male. Furthermore, the association phase of Experiment 2 involved only the between-speaker mapping, in which each object from critical item pairs was associated with a unique speaker. We focused on the between-speaker mapping condition because that is where we observed the critical speakerspecific effects in Experiment 1.

In addition, we also wanted to obtain evidence to bolster the claim that the critical effects observed in Experiment 1 were the result of relatively implicit access to the relevant speaker-item mappings - as assumed by the memory-based account outlined by Horton and Gerrig (2005a, b)-rather than being the result of participants' explicit recollections of which items were associated with which speaker. To this 
end, we asked participants at the end of the experimental session to carry out a speaker identification task in which they were asked to indicate, for each experimental item, whether it had been described by "Speaker A" or "Speaker B" during the association phase. If explicit access to relevant speaker-item mappings contributes to the degree to which listeners in the present paradigm are able to use speaker identity to anticipate reference, then participants with the strongest performance on the postexperimental speaker identification task should also show the strongest effects of using speaker identity to guide fixations to the expected targets. If, however, explicit memory is not a primary means by which listeners are anticipating reference in this task, then performance on the speaker identification task may be mostly irrelevant to reference interpretation.

\section{Method}

\section{Participants}

Fifteen undergraduates participated in this experiment for course credit or monetary compensation. Three participants were excluded due to poor calibration of the eyetracker, leaving 12 participants for analysis. All participants were native English speakers.

\section{Materials}

The sets of experimental and filler displays were identical to those used in Experiment 1. For the prerecorded instructions, we replaced the sound files from the female speaker in Experiment 1 with a new set of recordings obtained from another male confederate. These recordings were created under the same conditions as those used previously, including the same scripted inclusion of hedges and other disfluencies in instructions presented on early trials.

The association phase of Experiment 2 was identical to the between-partner mapping condition of Experiment 1: Participants heard each speaker repeatedly refer to one item from each of the 16 experimental item pairs, with different items from the pair being mentioned across speakers. Instructions from each speaker were presented during separate blocks, with 64 trials in a block (32 experimental trials involving tangrams and 32 filler trials involving line drawings of familiar objects). Thus, each speaker was intended to become associated with one particular objectexpression mapping from critical item pairs (e.g., either cat that's sitting up or cat that's drinking milk).

As in Experiment 1, the test phase included 16 critical trials that presented both items from critical item pairs and 16 control trials that included an experimental item as the target together with a different-category competitor. There were also 32 filler trials that involved familiar object targets with both same-category and different-category distractors. Participants heard each speaker give instructions for half of the 64 test trials, and trials from each speaker were again randomly intermixed to force participants to attend to speaker identity on a trial-by-trial basis. Finally, half of the trials involved targets that had been previously identified by the same speaker (same speaker trials), whereas the remaining trials involved targets previously identified by the other speaker (different speaker trials).

For the postexperimental speaker identification task, we prepared booklets that contained the 32 experimental tangrams, presented four to a page. The tangrams were ordered randomly, with the constraint that both tangrams from a given item pair could not appear on the same page. Instructions on the first page of the booklet told participants that they should indicate, for each tangram, whether it had been described first by "Speaker A" or by "Speaker B." The instructions emphasized that we were only interested in which speaker initially had described each item, and that participants should not allow themselves to be confused if an item had also been described by the other speaker in subsequent blocks. Beside each tangram was a blank where participants could give their response.

\section{Procedure}

The procedure of Experiment 2 was largely identical to that of Experiment 1. The cover story again emphasized the fact that participants would be hearing instructions recorded independently by two previous naive speakers, who were referred to as "Speaker A" and "Speaker B," with Speaker A giving the first set of instructions. It was also mentioned that both speakers would be male. During the first two blocks of the experiment, participants simply followed instructions to click on one of the pictures on each trial. Before each subsequent block, participants were taken through a brief calibration procedure for the eyetracker, and the critical eye fixation data were obtained during the fifth and final block, which comprised the test phase. Each trial consisted of a central fixation point for $1.5 \mathrm{~s}$, followed by a preview of the two pictures for $2 \mathrm{~s}$, then a fixation point again for $2 \mathrm{~s}$, and finally the two pictures reappeared simultaneously with the onset of the auditory instructions. After participants selected a picture on each trial, the next trial began. After the test phase, the experimenter gave participants the speaker identification booklet and reminded them of the identities of "Speaker A" and "Speaker B." After completing this booklet, participants were debriefed fully. All participants indicated that they had believed that the spoken instructions were from naive participants. 


\section{Design}

All participants experienced the between-speaker mapping condition during the association phase. In the test phase, the current speaker's relationship to a previous targetexpression mapping (same-speaker vs. different-speaker) was a within-subjects factor. Each experimental item appeared as the target once during the test block, and items were counterbalanced to ensure that each item appeared in both conditions across versions.

\section{Results}

As in Experiment 1, we coded participants' eye fixations on critical trials as landing on the intended target of the speaker's referring expression, on the competitor object, or on neither location. Then, we calculated the proportions of total fixations to the intended target and to the competitor within three temporal windows, time-locked to the accompanying utterance in the same manner as described for Experiment 1: the baseline period, the predisambiguation period, and postdisambiguation period. Within each time region, we computed target advantage scores by subtracting the proportions of looks to the competitor (out of all looks during each time period) from the proportions of fixations to the target (again, out of all looks during each time period). Mean target advantage scores for each time period are presented in Fig. 5. Again, the critical period of interest is the predisambiguation region, which represents the time from the onset of the noun to the onset of the informative modifying information, adjusted to take into account the time needed for planning and execution of individual

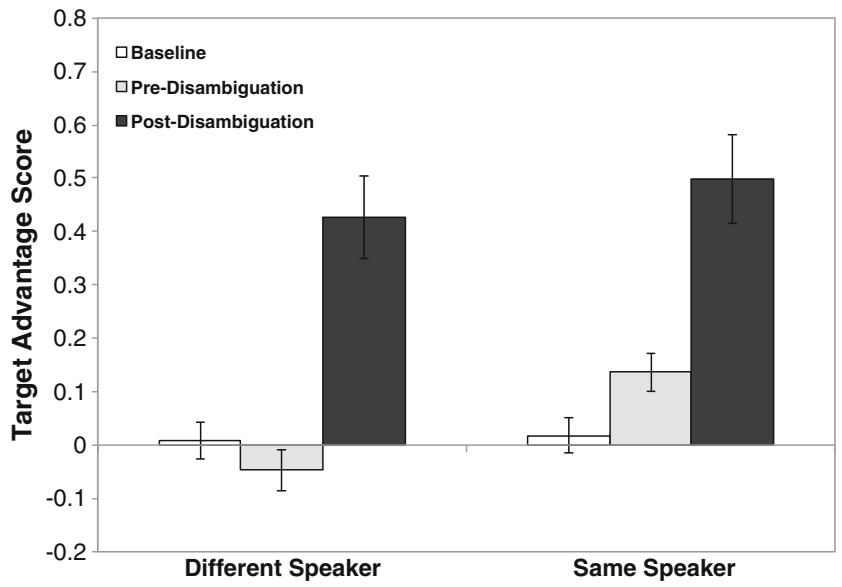

Fig. 5 Mean target advantage scores in Experiment 2, by speaker identity and analysis regions time locked to the accompanying speech. The target advantage score represents the proportion of total fixations to the target minus the proportion of total fixations to the competitor during each region. Error bars represent standard errors of the means for each group saccades. As Fig. 5 indicates, listeners on same partner trials were more likely to look at the target location during this time (and, to a lesser extent, more likely to look at the competitor location on different partner trials), suggesting that speaker identity was facilitating early expectations about the likely target of the current referring expression.

To verify this pattern, we carried out ANOVAs on target advantage scores for each time period separately, treating both subjects $\left(F_{1}\right)$ and items $\left(F_{2}\right)$ as random variables. In the baseline region, there were no differences between conditions (all $F \mathbf{s}<1$ ). In the critical predisambiguation region, however, there was a reliable effect of speaker identity, $F_{1}(1,11)=9.84$, $M S E=.020, p<.01 ; F_{2}(1,31)=7.75, M S E=.069, p<.01$, with listeners looking more strongly at targets on same-partner trials, $t_{1}(11)=3.81, p<.02 ; t_{2}(31)=2.53, p<.02$. Unlike in Experiment 1, however, listeners did not look significantly more to competitors on different partner trials during this time, $\left(t_{1}(11)=-1.20, p=.26 ; t_{2}(31)=-.92, p=.37\right.$. Finally, in the postdisambiguation region, listeners looked primarily at the target, with no difference between conditions (all $F_{\mathrm{s}}<1$ ).

To examine whether these effects could have been mediated at least in part by an explicit awareness of which speaker was associated with which item, we scored the data from the postexperimental speaker identification task. Across participants, correct speaker identification was significantly above chance $[M=67 \% ; t(11)=3.23, p<.01]$, with accuracy ranging from $25 \%$ to $88 \%$. Using a median split, we divided participants into "high accuracy" $(M=80 \%)$ and "low accuracy" $(M=54 \%)$ subgroups and assessed the extent to which each group, during the predisambiguation period, showed the critical pattern of differential attention to the target versus competitor according to speaker identity. Contrary to the notion that explicit recollection of speakeritem pairings may have been partially responsible for the overall pattern of speaker-specific anticipation of reference, only individuals in the low accuracy group - who on average were essentially at chance on the postexperimental identification task - showed a significant effect of speaker identity on target advantage scores during this period, $F_{1}(1,5)=8.48$, $M S E=.019, p<.04 ; F_{2}(1,15)=4.42, M S E=.098, p=$ .053 , with significantly more looks to the target on same speaker trials, $t_{l}(5)=2.96, p<.04 ; t_{2}(15)=2.36, p<.04$, and still no preference for the competitor on different speaker trials, $t_{l}(5)=-.82, p=.45 ; t_{2}(15)=-.39, p=.70$. In contrast, the target advantage scores during the predisambiguation period of individuals in the high accuracy group (who performed well at identifying the speaker-item pairings postexperimentally) failed to exhibit a reliable effect of speaker identity, $F_{1}(1,5)=2.34, M S E=.023, p=.19 ; F_{2}(1$, $15)=3.35, M S E=.043, p=.09$. Thus, the clearest evidence for speaker identity influencing reference resolution comes from those individuals who were least accurate in identifying the relevant speaker-specific mappings. 


\section{Discussion}

In Experiment 2, we largely replicated the critical results from Experiment 1. That is, despite the fact that both prerecorded speakers were of the same gender, the patterns of fixations to the target prior to the point of linguistic disambiguation indicate that speaker identity could function as a cue to allow listeners to anticipate reference. These results support a central claim of the memory-based account of conversational common ground, which is that the relevant constraints on language processing can be based on associations with respect to individual speakers and not just more general semantic categories such as gender. Even so, it is perhaps noteworthy that the locus of these speaker-specific effects in Experiment 2 comes primarily from the samespeaker trials, in which listeners showed an early preference for looking at target objects that were associated with the current speaker. Unlike in Experiment 1, in which listeners in the between-partner mapping condition also showed a preference for looking at the competitor on trials in which that competitor object was associated with the current speaker, the analogous effect for the different speaker trials of Experiment 2 was not significant. It is possible that having the additional cue of gender on top of the relevant speakerspecific associations strengthened the available memory traces in Experiment 1 in such a way that the early competitor advantage could be reliably observed on different speaker trials as well. Nevertheless, the results of Experiment 2 provide clear indication that speakers were prepared to look more strongly to the target object on trials in which the current speaker had described that object previously.

Moreover, the results of the postexperimental speaker identification task failed to support an alternative account of these speaker-specific effects as being mediated by explicit recall of relevant speaker-item mappings. Namely, the participants with the best explicit identification performance showed very weak and unreliable speaker-specific patterns of fixations to the target during the critical predisambiguation period. Instead, it was the participants who performed more or less at chance on the speaker identification task who showed the strongest evidence of using speaker identity as a cue about the likely target of the unfolding referring expression. Although it is possible that having more participants would lead to reliable effects in both groups, the overall pattern of weaker effects in the high-accuracy group argues against explanations for the critical speaker-specific effects as being rooted in relatively explicit memory processes.

\section{General discussion}

In two studies, we used a referent identification task to establish specific speaker-item memory associations for participants. In this association phase, participants repeatedly heard the same speaker use the same unique label when referring to a particular tangram. In Experiment 1, each speaker referred across trials to either one or both tangrams from particular item pairs belonging to the same semantic category, whereas in Experiment 2, each speaker was always uniquely associated with one item from a pair. Subsequently, in the test phase, we recorded participants' point of gaze to displays containing critical item pairs as they listened to temporarily ambiguous referring expressions. On these critical trials, we found that the nature of the initial memory associations facilitated the ability of listeners to anticipate the likely target of these expressions, but only in contexts in which the current speaker was associated with just one of the two available referents. Furthermore, in Experiment 2, we controlled the gender of the prerecorded speakers to rule out the possibility that these effects were due solely to the ability of listeners to encode critical mappings with respect to gender rather than to the identity of individual speakers. Although we believe that broader features associated with the conversational context, such as interlocutor gender, may represent important cues for the retrieval of relevant information in many circumstances, the fact that we were able to obtain evidence for speakerspecific anticipation in the absence of gender as a distinct cue suggests, at the very least, that participants in our paradigm were able to encode and have access to the relevant mappings with respect to particular speakers. Taken together, these results support the memory-based account proposed by Horton and Gerrig (2005a), which states that other individuals can function as salient cues to facilitate the retrieval of associated information from memory, and can do so in ways that function to implicitly constrain language processing to the relevant "common ground."

These results are similar to other work documenting the influence of talker-specific information on language processing. For example, Creel and colleagues (Creel, Aslin, \& Tanenhaus, 2008; Creel \& Tumlin, 2009) have shown that talker-specific characteristics can be used as predictive cues in spoken word recognition. Creel et al. (2008) trained listeners on phonological cohort word pairs (e.g., couch/ cows) spoken by the same or different talkers. Subsequently, words from different-talker pairs showed less lexical competition, as measured via eyetracking, than words from sametalker pairs. Thus, listeners appear to encode talker-based acoustic variation as part of their representations of lexical forms, consistent with models of spoken language processing in which word representations preserve traces of talkerspecific acoustic information (e.g., Goldinger, 1998). Presumably, such talker-specific acoustic traces form an important basis for the results observed in the present study. However, whereas Creel et al. examined phonological competition effects for single words, in the present study, we obtained effects of speaker identity given complex 
expressions for abstract tangrams, which generally elicit a broader range of referential forms. We chose to use tangrams on the expectation that doing so would highlight the speakerspecificity of any perspectives established for these abstract images (Clark \& Wilkes-Gibbs, 1986). Indeed, our results illustrate how these speaker-specific perspectives may be encoded into memory in such a way as to influence listeners' subsequent processing of temporarily ambiguous expressions produced by the same speakers.

However, it also appears that these speaker-specific encodings may be relatively fragile, at least in the context of our experimental paradigm. In Experiment 1, after experiencing critical trials in which speakers sometimes referred to the picture in the display other than the one with which they had been previously associated, listeners no longer reliably used speaker identity to anticipate reference. Although participants gave little indication of being overly aware of the incongruous trials during the final block, it is possible that simply providing unexpected mappings for given speaker-picture pairings may have weakened the existing mappings for the alternative referents from the same pairs. Whether this represents a case of generalization across item pairs or an item-by-item weakening of relevant associations is something that awaits further work.

An important claim of the memory-based account outlined by Horton and Gerrig (2005a) is that these speaker-specific constraints emerge in part on the basis of relatively implicit memory processes. That is, this account describes how language processing can be shaped by the cue-driven retrieval of information in ways that do not require access to explicit beliefs about common ground. In Experiment 2, we provided evidence to support this view, given that better performance on a task measuring explicit recall of the relevant speaker-item mappings was not associated with stronger evidence for speaker-specific anticipation effects. Indeed, it was the participants who were least able to explicitly recall these mappings who showed more of an impact of these associations on online reference interpretation. The apparent implicit nature of these effects is consistent with other demonstrations of memory-based effects on language comprehension (Myers \& O’Brien, 1998; Rawson, 2004) and also with other accounts suggesting that important aspects of interactive language use can be mediated through simple processes of memory accessibility and priming (Ferreira \& Dell, 2000; Pickering \& Garrod, 2004).

In the present context, we believe that each speaker's voice, together with the visually presented referents, likely served as a compound cue (McKoon \& Ratcliff, 1992; Ratcliff \& McKoon, 1988) for the implicit retrieval of relevant referentlabel mappings. In this respect, these results support the conclusions from Shintel and Keysar (2007), who found that listeners were just as "surprised" by unexpected referring expressions in situations when the expected expressions had been mutually established with a live speaker as when listeners had simply seen a video of the speaker. To explain these results, Shintel and Keysar distinguished between partner specificity, which involves sensitivity to the information mutually established between partners, and speaker specificity, which involves sensitivity to information associated with a given speaker, without regard to whether that information is mutually known. As Shintel and Keysar stated, speaker specificity implies that "the speaker's identity....can act as a cue even for expression-object mappings that are not assumed to be mutually known" (p. 368). The present work provides additional evidence for such speaker-specific processing, with the results of Experiment 2 suggesting further that this processing can happen on the basis of associations with respect to individual speakers, and not solely on the basis of more general social categories such as gender (although such social categories are presumably highly useful cues as well; Senay \& Keysar, 2009; van Berkum, van den Brink, Tesink, Kos, \& Hagoort, 2008).

However, unlike Shintel and Keysar (2007), speakers in the present study always used familiar names for referents on critical trials - what varied was whether speakers used names that only they had used previously. This critical difference may be why we were able to find evidence for speaker specificity using prerecorded instructions, contradictory to Brown-Schmidt (2009), who suggested that one reason why some studies have failed to show partner-specific effects is that they have relied on noninteractive situations. The potential conflict between two temporarily ambiguous expressions, each uniquely associated with a particular speaker in the context of a specific referent, may have been sufficient to allow the critical difference between speaker conditions to emerge following the between-speaker mapping, despite the fact that these mappings were established noninteractively.

At the same time, however, it should also be noted that these results are not completely consistent with the linguistic precedent account proposed by Barr and Keysar (2002) and Kronmüller and Barr (2007). In the present experiments, participants experienced identical numbers of referent-label mappings for all items during the association phase. On the simplest precedent account, then, participants should not have had strong early preferences for particular referents on critical trials, given that similar precedents were available for both items. Indeed, in the within-speaker mapping condition of Experiment 1, when both items were associated with the same speaker, this is what happened: Participants waited for sufficient input before looking strongly to the target. In the between-speaker mapping condition of Experiment 1 and in Experiment 2, however, participants appeared to rely on unique speaker-item associations to anticipate reference, suggesting that speaker identity acted as an additional constraint to direct initial attention toward associated pictures. 
In general, then, these results illustrate how speakerspecific memory associations may function as an important constraint upon online language interpretation. Whereas in previous work we documented the influence of memorybased processing on audience design and language production (Horton, 2007; Horton \& Gerrig, 2005b), in the present study, we have focused on the role these processes play in the moment-by-moment comprehension of language. Overall, to the extent that interlocutors serve as salient cues for the retrieval of specific patterns of relevant information, listeners appear to be able to use that information to anticipate the likely meanings of utterances. Moreover, if the circumstances are such that listeners have access to sufficiently distinct memory representations, this anticipation can happen relatively early, even before utterance meanings are fully available linguistically. In this manner, basic mechanisms of memory encoding and retrieval can routinely have an impact on higher level aspects of language use and social interaction.

Author Note This material is based on work supported by the National Science Foundation under Grant IIS-0705901. We wish to thank Robert Gunderson, Binoy Bhansali, and Jesse Sparks for their talents as confederate speakers, and Nicole Fortuna, Kyle Frost, and Vijay Singh for their help with stimulus creation and data collection. We also thank Katherine Rawson and three anonymous reviewers for their helpful feedback on an earlier version of this work.

\section{Appendix}

Table 1 List of expressions used to refer to each experimental item pair

the woman with the pointy butt the woman with the thin waist

the rabbit that's sitting down

the turtle with the tail

the rabbit that has two ears

the vulture with its head up

the square with the diamond

the triangle with the circle

the knife with the triangle on top

the Indian who's skating

the house with the garage

the candle with the large flame

the cat that's drinking milk

the bird that's pecking

the man who's running

the shark with no fin on top

the boat with the smokestack

the watering can with the long spout

Critical points of disambiguation are underlined

\section{References}

Barr, D. J., \& Keysar, B. (2002). Anchoring comprehension in linguistic precedents. Journal of Memory and Language, 46, 391-418.

Brennan, S. E., \& Clark, H. H. (1996). Conceptual pacts and lexical choice in conversation. Journal of Experimental Psychology. Learning, Memory, and Cognition, 22, 1482-1493.

Brown-Schmidt, S. (2009). Partner-specific interpretation of maintained referential precedents during interactive dialog. Journal of Memory and Language, 61, 171-190.

Clark, H. H. (1996). Using language. Cambridge: Cambridge University Press.

Clark, H. H., \& Carlson, T. B. (1981). Context for comprehension. In J. Long \& A. Baddeley (Eds.), Attention and performance IX (pp. 313-330). Hillsdale: Erlbaum.

Clark, H. H., \& Wilkes-Gibbs, D. (1986). Referring as a collaborative process. Cognition, 22, 1-39.

Creel, S. C., Aslin, R. N., \& Tanenhaus, M. K. (2008). Heeding the voice of experience: The role of talker variation in lexical access. Cognition, 106, 633-664.

Creel, S. C., \& Tumlin, M. A. (2009). Talker information is not normalized in fluent speech: Evidence from on-line processing of spoken words. Poster presented at the 31st Annual Conference of the Cognitive Science Society. Amsterdam, The Netherlands.

Ferreira, V. S., \& Dell, G. S. (2000). Effect of ambiguity and lexical availability on syntactic and lexical production. Cognitive Psychology, 40, 296-340.

Gerrig, R. J., \& McKoon, G. (2001). Memory processes and experiential continuity. Psychological Science, 12, 81-85.

Gillund, G., \& Schiffrin, R. M. (1984). A retrieval model for both recognition and recall. Psychological Review, 91, 1-67.

Goldinger, S. D. (1998). Echoes of echoes? An episodic theory of lexical access. Psychological Review, 105, 251-279.

Greene, S. B., Gerrig, R. J., McKoon, G., \& Ratcliff, R. (1994). Unheralded pronouns and management by common ground. Journal of Memory and Language, 33, 511-526.

Grossberg, S. (1980). How does the brain build a cognitive code? Psychological Review, 87, 1-51.

Horton, W. S. (2007). The influence of partner-specific memory associations on language production: Evidence from picture naming. Language and Cognitive Processes, 22, 1114-1139.

Horton, W. S., \& Gerrig, R. J. (2005a). Conversational common ground and memory processes in language production. Discourse Processes, 40, 1-35.

Horton, W. S., \& Gerrig, R. J. (2005b). The impact of memory demands on audience design during language production. Cognition, 96, 127-142.

Keysar, B., Barr, D. J., Balin, J. A., \& Brauner, J. S. (2000). Taking perspective in conversation: The role of mutual knowledge in comprehension. Psychological Science, 11, 32-38.

Kronmüller, E., \& Barr, D. J. (2007). Perspective-free pragmatics: Broken precedents and the recovery-from-preemption hypothesis. Journal of Memory and Language, 56, 436-455.

Lea, R. B., Mason, R. A., Albrecht, J. E., Birch, S. B., \& Myers, J. L. (1998). Who knows what about whom: What role does common ground play in accessing distant information? Journal of Memory and Language, 39, 70-84.

McKoon, G., \& Ratcliff, R. (1992). Spreading activation versus compound cue accounts of priming: Mediated priming revisited. Journal of Experimental Psychology. Learning, Memory, and Cognition, 18, 1155-1172.

Medin, D. L., \& Schaffer, M. M. (1978). Context theory of classification learning. Psychological Review, 85, 207-238. 
Metzing, C., \& Brennan, S. E. (2003). When conceptual pacts are broken: Partner-specific effects on the comprehension of referring expressions. Journal of Memory and Language, 49, 201-213.

Myers, J. L., \& O'Brien, E. J. (1998). Accessing the discourse representation during reading. Discourse Processes, 26, 131-157.

Myers, J. L., O’Brien, E. J., Albrecht, J. E., \& Mason, R. A. (1994). Maintaining global coherence during reading. Journal of Experimental Psychology. Learning, Memory, and Cognition, 20, 876-886.

Pickering, M. J., \& Garrod, S. (2004). Toward a mechanistic psychology of dialogue. Behavioral and Brain Sciences, 27, 169-226.

Ratcliff, R. (1978). A theory of memory retrieval. Psychological Review, 85, 59-108.

Ratcliff, R., \& McKoon, G. (1988). A retrieval theory of priming in memory. Psychological Review, 95, 385-408.

Rawson, K. A. (2004). Exploring automaticity in text processing: Syntactic ambiguity as a test case. Cognitive Psychology, 49, 333-369.
Senay, I., \& Keysar, B. (2009). Keeping track of speaker's perspective: The role of social identity. Discourse Processes, 46, 401-425.

Shintel, H., \& Keysar, B. (2007). You said it before and you'll say it again: Expectations of consistency in communication. Journal of Experimental Psychology. Learning, Memory, and Cognition, 33, 357-369.

Trabasso, T., \& Wiley, J. (2009). What happens at reunions? Exploring causal connections and their role in reunion effects. Discourse Processes, 46, 269-308.

Van Berkum, J. J. A., van den Brink, D., Tesink, M. J. Y. C., Kos, M., \& Hagoort, P. (2008). The neural integration of speaker and message. Journal of Cognitive Neuroscience, 20, 580-591.

Van der Wege, M. M. (2009). Lexical entrainment and lexical differentiation in reference phrase choice. Journal of Memory and Language, 60, 448-463. 